\title{
Subjective sleep complaints indicate objective sleep problems in psychosomatic patients: a prospective polysomnographic study
}

This article was published in the following Dove Press journal:

Nature and Science of Sleep

II August 2016

Number of times this article has been viewed

\author{
Michael Linden ${ }^{1,2}$ \\ Marie Dietz' \\ Christian Veauthier ${ }^{3}$ \\ Ingo Fietze ${ }^{3}$
}

'Research Group Psychosomatic Rehabilitation, Charité University Medicine Berlin, ${ }^{2}$ Department of Psychosomatic Medicine, Rehabilitation Centre Seehof, Teltow, ${ }^{3}$ Interdisciplinary Center of Sleep Medicine, Charité University Medicine Berlin, Berlin, Germany
Correspondence: Michael Linden Research Group Psychosomatic Rehabilitation, Charité University Medicine Berlin, CBF, Hs II, EOI, Hindenburgdamm 30, 12200 Berlin, Germany

$\mathrm{Tel} / \mathrm{fax}+493084454048$

Email michael.linden@charite.de
Objective: To elucidate the relationship between subjective complaints and polysomnographical parameters in psychosomatic patients.

Method: A convenience sample of patients from a psychosomatic inpatient unit were classified according to the Pittsburgh Sleep Quality Index (PSQI) as very poor sleepers (PSQI >10, $\mathrm{n}=80$ ) and good sleepers (PSQI $<6, \mathrm{n}=19$ ). They then underwent a polysomnography and in the morning rated their previous night's sleep using a published protocol (Deutschen Gesellschaft für Schlafforschung und Schlafmedizin morning protocol [MP]).

Results: In the polysomnography, significant differences were found between very poor and good sleepers according to the PSQI with respect to sleep efficiency and time awake after sleep onset. When comparing objective PSG and subjective MP, the polysomnographical sleep onset latency was significantly positively correlated with the corresponding parameters of the MP: the subjective sleep onset latency in minutes and the subjective evaluation of sleep onset latency (very short, short, normal, long, very long) were positively correlated with the sleep latency measured by polysomnography. The polysomnographical time awake after sleep onset (in minutes) was positively correlated with the subjective time awake after sleep onset (in minutes), evaluation of time awake after sleep onset (seldom, normal often), and subjective restfulness. The polysomnographical total sleep time (TST) was positively correlated with the subjective TST. Conversely, the polysomnographical TST was negatively correlated with the evaluation of TST (high polysomnographical TST was correlated with the subjective evaluation of having slept short or normal and vice versa). The polysomnographical sleep efficiency was positively correlated with subjective feeling of current well-being in the morning and subjective TST and negatively with subjective restfulness, subjective sleep onset latency, subjective evaluation of sleep onset latency, and evaluation of time awake after sleep onset.

Conclusion: The data suggest that, in general, patients selected from the extremes of reported very poor sleepers and good sleepers have different amounts of sleep when measured in the laboratory, and that in general, the amount and timing of sleep in the laboratory are quite well perceived and reported by these groups. The data came from psychosomatic patients and suggest that even in this patient group, respective sleep complaints are more than just the expression of general somatization or lamenting.

Keywords: Pittsburgh Sleep Quality Index, subjective sleep parameters, insomnia, awake after sleep onset, sleep disorders, sleep latency

\section{Introduction}

There is still a debate in the literature about the relationship between subjective sleep complaints and objective sleep parameters, measured by polysomnography. Some studies have found significant positive correlations between subjective estimates and objective 
polysomnographic variables such as total sleep time (TST) and sleep efficiency (SE). ${ }^{1,2}$ Still, a general notion is that subjective reports about sleep have only limited validity and are rather the result of sleep-related attitudes than real sleep problems. ${ }^{3}$ Several studies have shown that healthy subjects on the one hand tend to overestimate the latency to fall asleep, while on the other hand underestimate the durations of wakefulness during the night. ${ }^{3,4}$ Healthy volunteers overestimate their SE, while older patients with insomnia have a tendency to underestimate SE. ${ }^{5,6}$ Patients with insomnia tend to show a large discrepancy between subjective and objective sleep data.,8 Complaining poor sleepers even had a better objective sleep quality than noncomplaining good sleepers. ${ }^{8}$ Discrimination between good and poor sleepers is even more difficult in patients with comorbid disorders. Complaints about poor sleep and fatigue are regular symptoms of many somatic illnesses, be they neurological, internal, or orthopedic..$^{9-14}$ In these cases, it is difficult to discriminate between sleep complaints being a primary problem or being secondary to the somatic illness. This problem is even greater in psychosomatic patients as these patients have an increased tendency to see things in a negative light and to complain about their well-being and their sleep. ${ }^{15}$ Complaints about poor sleep are listed in the diagnostic criteria for many mental disorders, similar to others, the so-called "unexplained somatic symptoms", 3,16-25

Since reports of poor sleep in psychosomatic patients could be reflections of lamentation or somatization and/or poor perception rather than actual poor sleep, the aim of the present study was to investigate the extent to which subjective reports of poor sleep by psychosomatic patients have validity when compared to sleep measured in the laboratory.

\section{Methods}

\section{Participants}

This study has been reviewed and approved by the data protection department and the internal review board of the Federal German Pension Agency and all participants provided written informed consent.

This study was conducted between 2011 and 2012. In the Department of Psychosomatic Medicine, Rehabilitation Centre Seehof, Teltow/Berlin, Germany, all patients routinely fill in the Pittsburgh Sleep Quality Index (PSQI), ${ }^{9,26}$ which is an instrument to assess overall sleep quality. ${ }^{26}$ In this study, only 19 self-rating items were used. From a total of 1,325 patients, 92 were selected because of PSQI values $>10$ (very poor sleepers) and 22 because of PSQI values $<6$ (good sleepers). Ninety-nine out of these 114 patients agreed to participate in this study (PSQI $>10, n=80 / P S Q I<6, n=19$ ). Patients with ambiguous PSQI values between 6 and 10 were not enrolled in this study.
Patients were on average 48.04 years (standard deviation $[\mathrm{SD}]=9.0$; range: $19-87$ years) old, and $64.8 \%$ were female. Clinical diagnoses were affective disorders (International Classification of Diseases ${ }^{27}$ [ICD-10] F3) in $38.7 \%$, anxiety and somatoform disorders (ICD-10 F4) in $34.5 \%$, personality disorders (ICD-10 F6) in 9.9\%, developmental disorders (ICD-10 F7-9) in 5.3\%, substance abuse disorders (ICD-10 F1) in 5.2\%, eating disorders (ICD-10 F5) in 3.3\%, schizophrenic disorders (ICD-10 F2) in $2.6 \%$, and dementia (ICD-10 F0) in $2.6 \%$. The primary diagnosis of a sleep disorder was given by the treating physician in only $0.43 \%$.

\section{Assessment}

The PSQI is an instrument to assess overall sleep quality. ${ }^{26}$ In this study, only 19 self-rating items were used. Scores of $>10$ represent very poor sleepers and $<6$ good sleepers.

\section{Polysomnography}

After an adaptation night, a polysomnography was performed according to the American Academy of Sleep Medicine (AASM) standards, including electroencephalography (F3, F4, C3, C4, O1, O2, M1, M2), electrooculogram (vertical and horizontal eye movements), bipolar submentalis chin electromyogram, right and left bipolar anterior tibial muscle electromyogram, body position, ambient light and cardiorespiratory recording (eletrocardiography), nasal airflow (pressure sensor), tracheal microphone, thoracic and abdominal respiratory movements (piezo), and pulse oximetry (SOMNOscreen $^{\mathrm{TM}}$; SOMNOmedics, Randersacker, Germany). Sleep stages were classified manually according to the AASM classification rules. ${ }^{27}$ Respiratory events (hypopnea, apnea, desaturation) were preclassified by SOMNOmedics software and visually corrected by human raters according to the AASM rules. ${ }^{27}$ Polysomnographical data were analyzed to derive the following objective variables: sleep onset latency (oSOL; expressed in minutes), sleep efficiency (oSE; expressed as percentage of time in bed [TiB]), total sleep time (oTST; expressed as percentage of TiB), awake after sleep onset (expressed as percentage of TiB [oWASO] and in minutes [otWASO]), slow wave sleep (oSWS; expressed as percentage of TiB), rapid eye movement (REM) sleep (oREM; expressed as percentage of TiB), and frequency of microarousals from sleep (oMA; expressed per hour sleep).

\section{Morning protocol}

Patients completed the morning sleep protocol from the German Society of Sleep Research and Sleep Medicine immediately after the polysomnography night. ${ }^{28}$ Briefly, 
they were asked to make a subjective rating of current well-being (depressed/happy, weary/fresh, tense/relaxed), restfulness of prior sleep (sRF; my sleep was very, rather, somewhat, rather not, mostly not, not at all restful), sleep onset latency (sSOL; how long did it take to fall asleep), their evaluation of sleep onset latency (sESOL; was the time to fall asleep very short, short, normal, long, very long), awake after sleep onset (sWASO; how often have you been awake?), their evaluation of awake after sleep onset (sEWASO; seldom, normal often), time awake after sleep onset (sTWASO; how long have you been awake?), total sleep time (sTST), and their evaluation of total sleep time (sETST; short, normal, long).

Table I Comparison of the polysomnographical results between good and very poor sleepers

\begin{tabular}{|c|c|c|c|c|}
\hline \multirow{2}{*}{$\begin{array}{l}\text { Objective } \\
\text { parameter }\end{array}$} & \multirow{2}{*}{$\begin{array}{l}\text { PSQI > I0 } \\
(\text { mean } \pm \text { SD) }\end{array}$} & \multirow{2}{*}{$\begin{array}{l}\text { PSQI <6 } \\
(\text { mean } \pm \text { SD) }\end{array}$} & \multicolumn{2}{|c|}{ Test statistics } \\
\hline & & & $T$ & $P$-value \\
\hline SOL (minutes) & $12.75 \pm 17.68$ & $9.05 \pm 7.36$ & 0.96 & $0.34 I$ \\
\hline TST (minutes) & $396.01 \pm 60.27$ & $424.37 \pm 45.21$ & 1.86 & 0.066 \\
\hline WASO (\%/TiB) & $22.68 \pm 10.2$ & $21.61 \pm 7.81$ & 0.41 & 0.680 \\
\hline TWASO (minutes) & $46.97 \pm 39.15$ & $31.39 \pm 22.37$ & 2.2 & 0.032 \\
\hline SE (\%) & $86.87 \pm 8.81$ & $91.67 \pm 4.4$ & 3.64 & 0.001 \\
\hline $\begin{array}{l}\text { Content REM } \\
\text { sleep }(\%)\end{array}$ & $19.04 \pm 6.11$ & $|9.08 \pm 3.6|$ & 0.04 & 0.972 \\
\hline $\begin{array}{l}\text { Content slow wave } \\
\text { sleep (\%) }\end{array}$ & $|4.34 \pm 5.1|$ & $16.30 \pm 4.11$ & -1.67 & 0.097 \\
\hline $\begin{array}{l}\text { Microarousal } \\
\text { (per hour) }\end{array}$ & $31.50 \pm 14.85$ & $33.48 \pm 18.33$ & 0.54 & 0.584 \\
\hline
\end{tabular}

Notes: Very poor sleepers: PSQI $>10, n=80$ patients. Good sleepers: PSQI $<6, n=19$ patients. Significant results are shown in bold ( $P<0.05$, one tailed).

Abbreviations: PSQI, Pittsburgh Sleep Quality Index; SD, standard deviation; SOL, sleep onset latency; TST, total sleep time; WASO, awake after sleep onset; TWASO, time awake after sleep onset; SE, sleep efficiency; REM, rapid eye movement.

\section{Statistical methods}

The results were expressed as mean and SD. Patients were classified into two subgroups by PSQI values ( $>10$ versus $<6$ ). Following an exploratory analysis of the data and after a (negative) check for normality of the underlying distributions, differences between the two subgroups regarding polysomnographic parameters findings were (univariately) analyzed using a onetailed $t$-test. Statistical significance was established at $P<0.05$.

Regarding the correlation between subjective sleep parameters (PSQI/morning protocol [MP]) and objective parameters (polysomnographical), Kendall's Tau rank correlation coefficients were calculated. The level of significance was set at $P<0.001$ (two tailed). All tests were understood as constituting exploratory data analysis, such that neither previous power calculations nor subsequent adjustments for multiple testing had been made.

\section{Results}

\section{Polysomnographical results for good and poor sleepers}

Table 1 shows that very poor sleepers, as defined by the PSQI, have a significantly reduced polysomnographical-measured SE $(P=0.001)$ and longer time awake after sleep onset $(P=0.032)$ in comparison to those of good sleepers. There were no significant differences between these groups with regard to oSOL, oTST, oWASO, oREM, oSWS, and oMA.

\section{Correlations between subjective and objective sleep parameters}

Table 2 shows the correlations between subjective and objective sleep parameters for all patients $(\mathrm{N}=99)$. Significant

Table 2 Correlation between subjective and objective sleep parameters

\begin{tabular}{|c|c|c|c|c|c|c|c|c|c|c|c|c|}
\hline \multirow{3}{*}{$\begin{array}{l}\text { Objective } \\
\text { parameter }\end{array}$} & \multicolumn{12}{|c|}{ Subjective parameter } \\
\hline & \multicolumn{3}{|c|}{ Condition next morning } & \multirow[t]{2}{*}{ sRF } & \multirow[t]{2}{*}{ sSOL } & \multirow[t]{2}{*}{ sESOL } & \multirow[t]{2}{*}{ sWASO } & \multirow[t]{2}{*}{ sEWASO } & \multirow[t]{2}{*}{ sTWASO } & \multirow[t]{2}{*}{ sETWASO } & \multirow[t]{2}{*}{ sTST } & \multirow[t]{2}{*}{ sETST } \\
\hline & $\begin{array}{l}\text { Oppressed- } \\
\text { unburdened }\end{array}$ & $\begin{array}{l}\text { Weak- } \\
\text { bright }\end{array}$ & $\begin{array}{l}\text { Tensed- } \\
\text { relaxed }\end{array}$ & & & & & & & & & \\
\hline OSOL & -0.085 & -0.031 & -0.047 & 0.096 & $0.336 * *$ & $0.173 *$ & 0.160 & 0.061 & 0.160 & 0.065 & 0.065 & 0.019 \\
\hline oTST & 0.054 & 0.048 & 0.047 & 0.077 & 0.171 & 0.026 & 0.028 & 0.158 & 0.028 & 0.023 & $0.383^{* *}$ & $0.209 *$ \\
\hline oWASO & 0.047 & 0.045 & 0.124 & 0.185 & 0.183 & 0.066 & 0.021 & 0.183 & 0.021 & 0.194 & 0.097 & 0.042 \\
\hline oTWASO & 0.087 & 0.103 & 0.185 & $0.336 * *$ & 0.240 & 0.181 & $0.317^{*}$ & 0.023 & $0.317^{*}$ & $0.262 *$ & 0.166 & 0.075 \\
\hline oSE & 0.152 & 0.143 & $0.176 *$ & $0.239 * *$ & $0.36 \mathrm{I} * *$ & $0.246 * *$ & 0.074 & 0.048 & 0.074 & $0.223^{*}$ & $0.262 *$ & 0.047 \\
\hline oMA & 0.064 & 0.010 & 0.026 & 0.046 & 0.019 & 0.020 & 0.014 & 0.195 & 0.014 & 0.047 & 0.035 & 0.014 \\
\hline oREM & 0.082 & 0.143 & 0.073 & 0.049 & 0.134 & 0.051 & 0.056 & 0.084 & 0.056 & 0.005 & 0.065 & 0.050 \\
\hline oSWS & 0.093 & 0.000 & 0.081 & 0.066 & 0.164 & 0.206 & 0.021 & 0.074 & 0.021 & 0.119 & 0.047 & 0.005 \\
\hline
\end{tabular}

Notes: Significant results are highlighted in bold $(n=99, * P<0.005, * * P<0.001$, two tailed).

Abbreviations: sRF, subjective restfulness; sSOL, subjective sleep onset latency; sESOL, subjective evaluation of SOL; sWASO, subjective awake after sleep onset; sEWASO, subjective evaluation of awake after sleep onset; sTWASO, subjective time awake after sleep onset; sETWASO, subjective evaluation of time awake after sleep onset; sTST, subjective total sleep time; sETST, subjective evaluation of total sleep time; oSOL, objective sleep onset latency; oTST, objective total sleep time; oWASO, objective awake after sleep onset; oTWASO, objective time awake after sleep onset; oSE, objective sleep efficiency; oMA, objective microarousal; oREM, objective rapid eye movement sleep; oSWS, objective slow wave sleep. 
correlations were found between oSOL and $\operatorname{sSOL}(t=0.336$, $P<0.001)$ and sESOL $(t=0.173, P<0.005)$; oTST was correlated with $\operatorname{sTST}(t=0.383, P<0.001)$ and $\operatorname{sETST}(t=0.209$, $P<0.001)$; oTWASO was correlated with sRF $(t=0.336$, $P<0.001)$, sWASO $(t=0.317, P<0.005)$, sTWASO $(t=0.223$, $P<0.005)$, and sTST $(t=0.262, P<0.005)$; and oSE was correlated with subjective feeling of being relaxed in the morning $(t=0.176, P<0.005), \operatorname{sRF}(t=0.239, P<0.001), \operatorname{sSOL}(t=0.361$, $P<0.001)$, sESOL $(t=0.246, P<0.001)$, evaluation of time awake after sleep onset (sETWASO; $t=0.223, P<0.005$ ), and $\operatorname{sTST}(t=0.262, P<0.005)$.

\section{Discussion}

A main finding of this study is that in patients with psychosomatic disorders, reportedly very poor sleepers (with high PSQI scores) show objectively reduced oSE and an increased oTWASO compared to reportedly good sleepers (with low PSQI scores). These group trends suggest that the PSQI may be a useful diagnostic screening tool to screen for sleep disturbances even in psychosomatic patients. Nonetheless, in this patient population, trait insomnia seems to be related to problems of sleep maintenance and less to sleep initiation, while the amount of deep sleep has not been significantly different in very poor sleepers compared to good sleepers. This finding corresponds with previous findings. ${ }^{1,5}$ It seems possible that comorbid disorders, such as depression, anxiety, or pain, could help to explain this pattern.

A second main finding is that there are specific relations between oSOL and sSOL; between oSE and sRF, TST, SOL, and WASO; and between oWASO and sWASO and TST. In particular, data collected using the Deutschen Gesellschaft für Schlafforschung und Schlafmedizin MP correlate with the polysomnographical data (across these extreme groups in terms of PSQI scores), suggesting that the MP may be a useful diagnostic tool even in psychosomatic patients.

Our results may seem somewhat axiomatic since it seems likely that most people would be able to remember to some extent how long it took to fall asleep and whether there were repeated periods of awakening during the night. 1,2,29 However, in contrast to being awake, the macrostructure of sleep, including proportions of sleep stages, and the microstructure of sleep, including frequency of arousals, would seem to be less salient regarding the subjective experience of sleep. In sum, these correlations are meaningful and suggest that specific sleep complaints of patients reflect some underlying objective sleep problems even in psychosomatic patients. We suggest that sleep complaints should therefore be taken seriously and not played down as "just" complaints in such patients.

\section{Limitations}

The most important limitation is that PSQI scores were not normally distributed since we preselected patients from the two extremes. This distribution may have increased the likelihood of finding the correlations in the group data that emerged. In addition, the number of patients does not allow separate analyses for specific diagnostic subgroups. Furthermore, the analyses are cross-sectional and could vary in longitudinal observations.

\section{Conclusion}

The PSQI is a useful screening tool for insomnia in psychosomatic patients. Poor sleepers according to the PSQI in this specific patient population also had objective signs of poor sleep maintenance in the polysomnography and less problem with delayed initiation of sleep. Finally, subjective complaints of poor sleep in psychosomatic patients should be taken as indicators for objective sleep problems rather than as an expression of general lamenting.

\section{Disclosure}

The authors report no conflicts of interest in this work.

\section{References}

1. Kushida CA, Chang A, Gadkary C, Guilleminault C, Carrillo O, Dement WC. Comparison of actigraphic, polysomnographic, and subjective assessment of sleep parameters in sleep-disordered patients. Sleep Med. 2001;2(5):389-396.

2. Shochat T, Tzischinsky O, Oksenberg A, Peled R. Validation of the Pittsburgh Sleep Quality Index Hebrew translation (PSQI-H) in a sleep clinic sample. Isr Med Assoc J. 2007;9(12):853-856.

3. Baker F, Maloney S, Driver H. A comparison of subjective estimates of sleep with objective polysomnographic data in healthy men and women. J Psychosom Res. 1999;47(4):335-341.

4. Lauderdale DS, Knutson KL, Yan LL, et al. Objectively measured sleep characteristics among early-middle-aged adults: the CARDIA study. Am J Epidemiol. 2006;164(1):5-16.

5. Gooneratne N, Bellamy S, Pack F, et al. Case-control study of subjective and objective differences in sleep patterns in older adults with insomnia symptoms. J Sleep Res. 2011;20(3):434-444.

6. Unruh M, Redline S, An MW, et al. Subjective and objective sleep quality and aging in the sleep heart health study. JAm Geriatr Soc. 2008;56(7): 1218-1227.

7. McCall WV, Turpin E, Reboussin D, Edinger JD, Haponik EF. Subjective estimates of sleep differ from polysomnographic measurements in obstructive sleep apnea patients. Sleep. 1995;18(8):646-650.

8. McCrae CS, Rowe MA, Tierney CG, Dautovich ND, Definis AL, McNamara JP. Sleep complaints, subjective and objective sleep patterns, health, psychological adjustment, and daytime functioning in community-dwelling older adults. J Gerontol B Psychol Sci Soc Sci. 2005;60(4):182-189. 
9. Fictenberg NL, Putnam SH, Mann NR, Zafonte RD, Millard AE. Insomnia screening in postacute traumatic brain injury: utility and validity of the Pittsburgh Sleep Quality Index. Am J Phys Med Rehabil. 2001;80(5):339-345.

10. Mbatchou Ngahane BH, Nganda MM, Dzudie A, et al. Prevalence and determinants of excessive daytime sleepiness in hypertensive patients: a cross-sectional study in Douala, Cameroon. BMJ Open. 2015;5(7): e008339.

11. Veauthier C. Poor versus good sleepers in patients under treatment for sleep-related breathing disorders: better is not good enough. Neuropsychiatr Dis Treat. 2014;10:131-133.

12. Milewski MD, Skaggs DL, Bishop GA, et al. Chronic lack of sleep is associated with increased sports injuries in adolescent athletes. J Pediatr Orthop. 2014;34(2):129-133.

13. Ismayilova V, Demir AU, Tezer FI. Subjective sleep disturbance in epilepsy patients at an outpatient clinic: a questionnaire-based study on prevalence. Epilepsy Res. 2015;115:119-125.

14. Sands SA, Owens RL. Congestive heart failure and central sleep apnea. Crit Care Clin. 2015;31(3):473-495.

15. Sivertsen B, Krokstad S, Øverland S, Mykletun AJ. The epidemiology of insomnia: associations with physical and mental health. The HUNT-2 study. J Psychosom Res. 2009;67(2):109-116.

16. Annagür BB, Uguz F, Apiliogullari S, Kara I, Gunduz S. Psychiatric disorders and association with quality of sleep and quality of life in patients with chronic pain: a SCID-based study. Pain Med. 2014;15(5):772-781.

17. Argyropoulos S, Hicks J, Nash J, et al. Correlation of subjective and objective sleep measurements at different stages of the treatment of depression. Psychiatry Res. 2003;120(2):179-190.

18. Leblanc MF, Desjardins S, Desgagné A. Sleep problems in anxious and depressive older adults. Psychol Res Behav Manag. 2015;8:161-169.

19. Linden M. Institutionelle Prävalenz und Kontext schwerer Schlafstörungen bei Patienten in psychosomatischer Rehabilitation. Psychother Psych Med. 2015;65(7):268-272.
20. Linder J, Jansen GB, Ekholm KS, Ekholm J. Relationship between sleep disturbance, pain, depression and functioning in long-term sick-listed patients experiencing difficulty in resuming work. $J$ Rehabil Med. 2014;46(8):798-805.

21. Murphy MJ, Peterson MJ. Sleep disturbances in depression. Sleep Med Clin. 2015;10(1):17-23.

22. Neckelmann D, Mykletun A, Dahl A. Chronic insomnia as a risk factor for developing anxiety and depression. Sleep. 2007;30(7):873-880.

23. Orff HJ, Drummond SP, Nowakowski S, Perils ML. Discrepancy between subjective symptomatology and objective neuropsychological performance in insomnia. Sleep. 2007;30(9):1205-1211.

24. Szelenberger W, Soldatos C. Sleep disorders in psychiatric practice. World Psychiatry. 2005;4(3):186-190.

25. WHO. International Statistical Classification of Diseases and Related Health Problems. 10 th Revision. Geneva: WHO; 1992.

26. Buysse D, Reynolds C, Monk T. Pittsburgh Sleep Quality Index: a new instrument for psychiatric practice and research. Psychiatry Res. 1989;28(2):193-213.

27. Berry RB, Brooks R, Gamaldo CE, et al; American Association of Sleep Medicine [homepage on the Internet]. The AASM Manual for the Scoring of Sleep and Associated Events: Rules, Terminology and Technical Specifications, Version 2.0. Darien, IL: American Academy of Sleep Medicine; 2012. Available from: www.aasmnet.org. Accessed September 27, 2015.

28. Hoffmann RM, Müller T, Hajak G, Cassel W; Arbeitsgruppe Diagnostik der Deutschen Gesellschaft für Schlafforschung und Schlafmedizin (DGSM). Abend-Morgen-Protokolle in Schlafforschung und Schlafmedizin - ein Standardinstrument für den deutschsprachigen Raum. Somnologie. 1997;1:103-109.

29. McCall C, McCall WV. Objective vs. subjective measurements of sleep in depressed insomniacs: first night effect or reverse first night effect? J Clin Sleep Med. 2012;8(1):59-65.
Nature and Science of Sleep

\section{Publish your work in this journal}

Nature and Science of Sleep is an international, peer-reviewed, open access journal covering all aspects of sleep science and sleep medicine, including the neurophysiology and functions of sleep, the genetics of sleep, sleep and society, biological rhythms, dreaming, sleep disorders and therapy, and strategies to optimize healthy sleep. The manuscript

\section{Dovepress}

management system is completely online and includes a very quick and fair peer-review system, which is all easy to use. Visit http://www. dovepress.com/testimonials.php to read real quotes from published authors. 